Claremont Colleges

Scholarship@ Claremont

All HMC Faculty Publications and Research

HMC Faculty Scholarship

3-1-1998

\title{
Onset of Superconductivity in Decreasing Fields for General Domains
}

\author{
Andrew J. Bernoff \\ Harvey Mudd College \\ Peter Sternberg \\ Indiana University - Bloomington
}

\section{Recommended Citation}

Onset of superconductivity in decreasing fields for general domains. Andrew Bernoff and Peter Sternberg, J. Math. Phys. 39, 1272 (1998).

This Article is brought to you for free and open access by the HMC Faculty Scholarship at Scholarship @ Claremont. It has been accepted for inclusion in All HMC Faculty Publications and Research by an authorized administrator of Scholarship @ Claremont. For more information, please contact scholarship@cuc.claremont.edu. 


\title{
Onset of superconductivity in decreasing fields for general domains
}

\author{
Andrew Bernoff ${ }^{a}$ \\ Department of Mathematics, Duke University, Durham, North Carolina 27708 \\ Peter Sternberg ${ }^{\text {b) }}$ \\ Department of Mathematics, Indiana University, Bloomington, Indiana 47405
}

(Received 10 September 1997; accepted for publication 12 November 1997)

Ginzburg-Landau theory has provided an effective method for understanding the onset of superconductivity in the presence of an external magnetic field. In this paper we examine the instability of the normal state to superconductivity with decreasing magnetic field for a closed smooth cylindrical region of arbitrary crosssection subject to a vertical magnetic field. We examine the problem asymptotically in the boundary layer limit (i.e., when the Ginzburg-Landau parameter, $k$, is large). We demonstrate that instability first occurs in a region exponentially localized near the point of maximum curvature on the boundary. The transition occurs at a value of the magnetic field associated with the half-plane at leading order, with a small positive correction due to the curvature (which agrees with the transition problem for the disc), and a smaller correction due to the second derivative of the curvature at the maximum. (C) 1998 American Institute of Physics.

[S0022-2488(98)00203-5]

\section{INTRODUCTION}

The application of a sufficiently large magnetic field is known to destroy the superconducting property of a superconductor. If one keeps a sample below some critical temperature, however, then experiments reveal the emergence of a superconducting state once the external field is lowered below some critical value. In addition to a loss of normal resistivity, the material in the superconducting state gains the ability to expel the applied magnetic field. Here we investigate this bifurcation using the Ginzburg-Landau theory of superconductivity as a model. ${ }^{1}$

This problem has been treated analytically in various settings in which the sample is cylindrical (i.e., of constant cross section), and the applied magnetic field points in the axial direction. This assumption allows for a two-dimensional approach to the analysis. Saint-James and de Gennes $^{2}$ consider the case of a half-plane and infinite slab, and using the Ginzburg-Landau theory they linearize the equations about the so-called "normal state" in which the applied field totally permeates the medium. Through an explicit solution, they find the critical value of the applied field where the normal state loses stability-frequently referred to as $H_{c_{3}}$ in the literature- to be approximately $1.7 k$. Here $k$ is the Ginzburg-Landau parameter, a dimensionless material constant defined as the ratio of two important length scales in the system. In their analysis and in ours as well, $k$ is taken to be greater than $1 / \sqrt{2}$, so that the material is a so-called type-II superconductor. Saint-James and de Gennes also find that the superconducting state concentrates along a thin neighborhood of the boundary of the sample and then tails off exponentially in the interior, a property referred to as "surface superconductivity." More recently, Chapman ${ }^{3,4}$ carried out a more detailed formal mathematical treatment of the half-plane problem as part of a general analysis of onset for decreasing fields, starting from a perturbation theory developed by Millman and Keller. ${ }^{5}$ (See also Refs. $6-8$ for related rigorous work and more recently, ${ }^{9}$ for rigorous estimates on the upper critical field, above which only normal states solve the Ginzburg-Landau equations.)

Following these studies, Bauman, Phillips, and Tang ${ }^{10}$ studied this bifurcation when the cross section of the sample is a disc. By separating variables and using a highly nontrivial O.D.E.

\footnotetext{
${ }^{a)}$ Electronic mail: ajb@nwu.edu

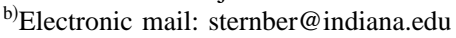


analysis, they rigorously show that the value of $H_{c_{3}}$ is raised above the half-plane value of $1.7 k$ by a quantity proportional to the curvature of the disc. As in the case of a half-plane, they find the superconducting state concentrates near the boundary of the disc with an exponentially small tail.

It was this last result which caught our attention and lead us to ask the question: what is the nature of the bifurcating superconducting state when the cross section of the sample has an arbitrary shape? Here we investigate this problem using formal asymptotic expansions in the regime where $k$ is large. We discover that the transition occurs at a value of the external field associated with the half-plane at leading order, with a small positive correction proportional to the maximum of curvature (which agrees with the transition problem for the disc), and a smaller correction due to the second derivative of the curvature at the maximum. Furthermore, the amplitude of the order parameter measuring the presence of superconductivity is exponentially localized about the point on the boundary where curvature is a maximum. We note that recently Chapman ${ }^{11}$ studied a complementary bifurcation problem in which a small applied magnetic field is gradually raised until it reaches a critical field $\left(H_{c_{1}}\right)$ at which the purely superconducting state loses stability to a vortex state. In that setting it turns out that the bifurcating vortex state emerges near the point of maximum curvature of the boundary as well.

In the section which follows we introduce the Ginzburg-Landau model and then present the asymptotic analysis leading to the conclusions stated above.

\section{ASYMPTOTIC ANALYSIS}

We consider a sample occupying an infinite cylinder with two-dimensional cross section consisting of an arbitrary simply connected smooth bounded region $\Omega \subset \mathbf{R}^{2}$. The sample is adjacent to a vacuum and is subjected to an applied magnetic field $\mathbf{H}$ pointing in the axial direction, so that $\mathbf{H}=H \hat{\mathbf{z}}$ for some positive scalar $H$. We use the Ginzburg-Landau theory to model the superconductor, thus the system is described by two quantities, an order parameter $\Psi: \Omega \rightarrow \mathbf{C}$ and the magnetic potential $\mathbf{A}: \Omega \rightarrow \mathbf{R}^{2}$. The pair $(\Psi, \mathbf{A})$ is an equilibrium state for the energy

$$
G(\Psi, \mathbf{A})=\int_{\Omega}\left(\frac{1}{2}\left|\left(\frac{i}{k} \nabla+\mathbf{A}\right) \Psi\right|^{2}+\frac{1}{4}\left(1-|\Psi|^{2}\right)^{2}+\frac{1}{2}|\operatorname{curl} \mathbf{A}-H \hat{\mathbf{z}}|^{2}\right) d x,
$$

where curl $\mathbf{A}=\left(\partial_{x} A_{2}-\partial_{y} A_{1}\right) \hat{\mathbf{z}}, k$ is the Ginzburg-Landau parameter and we have used the penetration depth as a length scale. This energy is well-defined for $\Psi$ belonging to the Sobolev space $H^{1}(\Omega ; C)$ and $\mathbf{A}$ belonging to the Sobolev space $H^{1}\left(\Omega ; \mathbf{R}^{2}\right)$. Setting the first variation of $G$ to zero, one finds that the pair $(\Psi, \mathbf{A})$ satisfies the Ginzburg-Landau equations

$$
\begin{gathered}
\left(\frac{i}{k} \nabla+\mathbf{A}\right)^{2} \Psi-\Psi+|\Psi|^{2} \Psi=0 \text { in } \Omega, \\
(\operatorname{curl})^{2} \mathbf{A}+\frac{i}{2 k}\left(\Psi^{*} \nabla \Psi-\Psi \nabla \Psi^{*}\right)+\left|\Psi^{2}\right| \mathbf{A}=0 \text { in } \Omega,
\end{gathered}
$$

along with the boundary conditions

$$
\begin{gathered}
\hat{\mathbf{n}} \cdot\left(\frac{i}{k} \nabla+\mathbf{A}\right) \Psi=0 \text { on } \partial \Omega, \\
\operatorname{curl} \mathbf{A}=H \hat{\mathbf{z}} \text { or } \partial \Omega .
\end{gathered}
$$

Here $\hat{\mathbf{n}}$ is the inward unit normal to $\partial \Omega$ and $(\cdot)^{*}$ denotes complex conjugation. We refer to Ref. 12 for more background on the model. We also note that in light of our assumption of smoothness on $\partial \Omega$, standard elliptic regularity theory shows that solutions to $(2.2)-(2.5)$ are classical $\left(C^{2}\right)$ solutions.

The problem is invariant under the gauge transformation $(\Psi, \mathbf{A}) \rightarrow\left(\Psi^{\prime}, \mathbf{A}^{\prime}\right)$ where

$$
\Psi^{\prime} \equiv \Psi e^{i k \phi}, \quad \mathbf{A}^{\prime} \equiv \mathbf{A}+\nabla \phi
$$


for an arbitrary real-valued $H^{2}(\Omega)$ function $\phi$. Throughout this analysis it will be convenient to impose the additional conditions

$$
\operatorname{div} \mathbf{A}=0 \text { in } \Omega, \mathbf{A} \cdot \hat{\mathbf{n}}=0 \text { on } \partial \Omega .
$$

This amounts to choosing a gauge, thus eliminating the degeneracy associated with the gauge invariance of our problem.

Within the Ginzburg-Landau model the normal state corresponds to the conditions

$$
\Psi \equiv 0, \quad \mathbf{A}=\overline{\mathbf{A}},
$$

where $\overline{\mathbf{A}}$ satisfies

$$
\begin{gathered}
(\operatorname{curl})^{2} \overline{\mathbf{A}}=0 \text { in } \Omega, \\
\operatorname{curl} \overline{\mathbf{A}}=H \hat{\mathbf{z}} \text { on } \partial \Omega, \\
\operatorname{div} \overline{\mathbf{A}}=0 \text { in } \Omega, \\
\overline{\mathbf{A}} \cdot \hat{\mathbf{n}}=0 \text { on } \partial \Omega .
\end{gathered}
$$

For a vector with only two components the condition (curl) ${ }^{2} \overline{\mathbf{A}}=0$ implies that curl $\overline{\mathbf{A}}$ is constant so the conditions above reduce to simply

$$
\begin{aligned}
& \operatorname{curl} \overline{\mathbf{A}}=H \hat{\mathbf{z}} \text { in } \Omega, \\
& \operatorname{div} \overline{\mathbf{A}}=0 \text { in } \Omega, \\
& \overline{\mathbf{A}} \cdot \hat{\mathbf{n}}=0 \text { on } \partial \Omega .
\end{aligned}
$$

It is convenient to introduce the vector field $\overline{\mathbf{a}}$ through the relation $\overline{\mathbf{A}}=H \overline{\mathbf{a}}$, so that

$$
\begin{aligned}
& \operatorname{curl} \overline{\mathbf{a}}=\hat{\mathbf{z}} \text { in } \Omega, \\
& \operatorname{div} \overline{\mathbf{a}}=0 \text { in } \Omega, \\
& \overline{\mathbf{a}} \cdot \hat{\mathbf{n}}=0 \text { on } \partial \Omega .
\end{aligned}
$$

We note that obtaining the existence of a classical solution to (2.8) in a smooth bounded domain is simple. For example, one can obtain $\overline{\mathbf{a}}$ in the form $\overline{\mathbf{a}}=\frac{1}{2}(-y, x)+\nabla u$, where $u$ is a harmonic function satisfying the Neumann condition $\nabla u \cdot \hat{\mathbf{n}}=-\frac{1}{2}(-y, x) \cdot \hat{\mathbf{n}}$ on $\partial \Omega$. The uniqueness of $\overline{\mathbf{a}}$ follows from the assumption of simple connectivity on $\Omega$. That is, letting say $\mathbf{b}$ denote the difference between any two solutions to (2.8), one finds that $\mathbf{b}$ must be the gradient of a harmonic scalar function whose normal derivative vanishes on $\partial \Omega$. Thus the scalar function is constant and $\mathbf{b} \equiv 0$.

Since we seek a description of the initial bifurcation of the solution from the normal state $(0, H \overline{\mathbf{a}})$ as the applied magnetic field is decreased, we begin our formal analysis by introducing a small parameter $\epsilon$ (which measures the magnitude of $|\Psi|$ ) and assuming asymptotic expansions for the quantities $\Psi, \mathbf{A}$, and $H$ of the form

$$
\begin{gathered}
\Psi=0+\epsilon \Psi^{(1)}+\cdots, \\
\mathbf{A}=h \overline{\mathbf{a}}+\epsilon^{2} \mathbf{A}^{(2)}+\cdots, \\
H=h+\epsilon^{2} H^{(1)}+\cdots
\end{gathered}
$$


(cf. Refs. 3 and 5). The relative scalings of $\Psi, \mathbf{A}$, and $H$ are motivated by the need to balance the term $|\Psi|^{2} \Psi$ with the terms involving $\mathbf{A}-H \overline{\mathbf{a}}$ in (2.2). Inserting these expansions into (2.2)-(2.5) yields a linear problem for $\Psi^{(1)}$ at leading order,

$$
\begin{gathered}
\left(\frac{i}{k} \nabla+h \overline{\mathbf{a}}\right)^{2} \Psi^{(1)}-\Psi^{(1)}=0 \text { in } \Omega, \\
\hat{\mathbf{n}} \cdot\left(\frac{i}{k} \nabla+h \overline{\mathbf{a}}\right) \Psi^{(1)}=0 \quad \text { on } \partial \Omega .
\end{gathered}
$$

As $h$ is decreased, one expects the instability of the normal state to occur when (2.9)-(2.10) has a nontrivial solution. Here, $h$ plays the role of an eigenvalue and we define $H_{c_{3}}$ to be the largest positive eigenvalue for the linearized problem (2.9)-(2.10). When $k$ is sufficiently large, nonlinear analysis suggests that this bifurcation is a supercritical pitchfork (cf. Refs. 3 and 4).

At this point, we wish to analyze (2.9)-(2.10) via formal matched asymptotic expansions in the regime where the Ginzburg-Landau parameter $k$ is large. Experiments (both physical and numerical) indicate that the superconducting state nucleates on the boundary of $\Omega$ and the factor of $(1 / k)$ in $(2.9)$ suggests that the order parameter $\Psi^{(1)}$ will vary there with a characteristic length scale $1 / k$. Thus, we will seek a boundary layer solution which matches to the normal state in the interior of the domain. (Indeed, we will find that the modulus of this boundary layer solution decays exponentially to zero as we depart from $\partial \Omega$.)

To this end, we introduce coordinates $(s, \eta)$ valid in a neighborhood of $\partial \Omega$. Here $s$ is arclength along $\partial \Omega$ and $\eta$ is the distance from a point within $\Omega$ to the boundary. We can arbitrarily select a point on $\partial \Omega$ corresponding to $s=0$; without loss of generality we will choose this point to coincide with the maximum of curvature on the boundary. We will use $\kappa=\kappa(s)$ to denote the curvature of $\partial \Omega$ (not to be confused with the Ginzburg-Landau parameter $k$ !).

This local coordinate system will be well-defined in the rectangle

$$
S \equiv\{(s, \eta):-L / 2<s<L / 2,0<\eta<C\},
$$

where $L$ denotes the arclength of the boundary, and $C$ is any positive constant smaller than $[1 / \kappa(0)]$. We adopt the convention that for $\Omega$ a disc $\kappa$ is positive. We denote by $\hat{\mathbf{t}}=\hat{\mathbf{t}}(s)$ a unit tangent vector to $\partial \Omega$ and we recall that $\hat{\mathbf{n}}=\hat{\mathbf{n}}(s)$ denotes the inner unit normal vector. Thus, in particular, any vector field $\mathbf{F}$ defined in a neighborhood of $\partial \Omega$ can be expressed as $\mathbf{F}(s, \eta)$ $=F_{1}(s, \eta) \hat{\mathbf{t}}(s)+F_{2}(s, \eta) \hat{\mathbf{n}}(s)$. We now proceed to rewrite problem (2.9)-(2.10) in $s-\eta$ coordinates so that we may pursue a boundary layer construction in the region $S$.

For this purpose, we record here that for any scalar-valued function $f=f(s, \eta)$ one has the identities,

$$
\begin{gathered}
\nabla f=\partial_{\eta} f \hat{\mathbf{n}}+\frac{1}{1-\kappa \eta} \partial_{s} f \hat{\mathbf{t}}, \\
\nabla^{2} f=\partial_{\eta \eta} f-\frac{\kappa}{1-\kappa \eta} \partial_{\eta} f+\frac{1}{1-\kappa \eta} \partial_{s}\left(\frac{1}{1-\kappa \eta}\right) \partial_{s} f,
\end{gathered}
$$

and for any vector field $\mathbf{F}=F_{1}(s, \eta) \hat{\mathbf{t}}+F_{2}(s, \eta) \hat{\mathbf{n}}$ we have

$$
\begin{gathered}
\operatorname{div} \mathbf{F}=\frac{1}{1-\kappa \eta} \partial_{s} F_{1}+\frac{1}{1-\kappa \eta} \partial_{\eta}\left[(1-\kappa \eta) F_{2}\right], \\
\operatorname{curl} \mathbf{F}=\operatorname{div}\left(F_{2} \hat{\mathbf{t}}-F_{1} \hat{\mathbf{n}}\right) \hat{\mathbf{z}} .
\end{gathered}
$$

These identities can be computed using standard planar differential geometry (see e.g., Ref. 13) and are explicitly derived for example in Ref. 14.

For a general domain $\Omega$, although $\overline{\mathbf{a}} \cdot \hat{\mathbf{n}}=0$ on $\partial \Omega, \overline{\mathbf{a}}$ is not purely tangential. That is, writing $\overline{\mathbf{a}}=\overline{\mathbf{a}}(s, \eta)$ we have $\overline{\mathbf{a}}(s, \eta) \cdot \hat{\mathbf{n}}(s)=0$ only for $\eta=0$ but not for $0<\eta<C$. However in the coordinate rectangle $S$, we can decompose $\overline{\mathbf{a}}$ into components $\mathbf{p}$ and $\mathbf{q}$, 


\section{$\overline{\mathbf{a}}=\mathbf{p}+\mathbf{q}$}

as follows. We first define $\mathbf{q}$ as the solution to the problem

$$
\operatorname{curl} \mathbf{q}=\hat{\mathbf{z}} \text { in } S, \mathbf{q} \cdot \hat{\mathbf{n}}=0 \text { in } S, \text { and } \mathbf{q}(s, 0)=0 .
$$

Note in particular that $\mathbf{q}$ will be purely tangential is the sense mentioned above.

Seeking a solution to (2.15) in the form $\mathbf{q}=q(s, \eta) \hat{\mathbf{t}}$, we can solve for the scalar $q$ through the use of (2.13) and (2.14). We find that $q$ must satisfy the first order differential equation

$$
\frac{-1}{1-\kappa \eta} \partial_{\eta}((1-\kappa \eta) q)=1, q(s, 0)=0,
$$

so that

$$
\mathbf{q}(s, \eta)=q(s, \eta) \hat{\mathbf{t}}(s)=-\eta\left(\frac{1-\kappa(s) \eta / 2}{1-\kappa(s) \eta}\right) \hat{\mathbf{t}}(s) .
$$

We then define $\mathbf{p}$ by the relation $\overline{\mathbf{a}}=\mathbf{p}+\mathbf{q}$. Note in particular that $\mathbf{p}$ will then be conservative,

$$
\text { curl } \mathbf{p}=0 \text { in } S \text {. }
$$

Motivated by the gauge invariance, and utilizing (2.17), we now introduce a phase $\Phi$ on the rectangle $S$ through the relation $\nabla \Phi=h \mathbf{p}$. Hence, for any $(s, \eta) \in S$, we let

$$
\Phi(s, \eta)=h \int_{\gamma} \mathbf{p} \cdot d \mathbf{r},
$$

where $\gamma$ is any path in $S$ joining $(0,0)$ to $(s, \eta)$. We then introduce the function $\psi$ by writing the solution $\Psi^{(1)}$ to (2.9)-(2.10) near $\partial \Omega$ in the form

$$
\Psi^{(1)}=\psi e^{i k \Phi} e^{i k \beta s},
$$

where $\beta$ is a constant to be determined. Through the gauge transformation it becomes apparent that the complex exponential of $\Phi$ eliminates $\mathbf{p}$, the conservative component of $\overline{\mathbf{a}}$. The factor $e^{i k \beta s}$ represents a contribution to the phase evident in the earlier investigations of Saint-James and de Gennes $^{2}$ and Chapman $^{3}$ that arises when the problem is posed on the half-plane. Our motivation for this change of variables is to make the leading-order balance conform with the known results for the half-plane problem. In particular this is not a polar decomposition; although $\psi$ will have real solutions at leading order, the higher-order corrections may be complex in nature.

Using (2.6), we find that in a neighborhood of $\partial \Omega$, the problem (2.9)-(2.10) for $\Psi^{(1)}$ as a function of $x$ transforms to the following problem for $\psi$ as a function of $s$ and $\eta$,

$$
\begin{gathered}
\left(\frac{i}{k} \boldsymbol{\nabla}+h \mathbf{q}-\beta \boldsymbol{\nabla} s\right)^{2} \psi-\psi=0 \text { in } S, \\
\hat{\mathbf{n}} \cdot\left(\frac{i}{k} \nabla+h \mathbf{q}-\beta \nabla s\right) \psi=0 \text { on } \eta=0 .
\end{gathered}
$$

Then using (2.11) to compute $\nabla s$ and exploiting the fact that $h \mathbf{q}-\beta \nabla s$ has no $\hat{\mathbf{n}}$ component, we see that $(2.20)-(2.21)$ can be written as

$$
\begin{gathered}
\left(\frac{i}{k} \nabla+\left(h q-\frac{\beta}{1-\kappa \eta}\right) \hat{\mathbf{t}}\right)^{2} \psi-\psi=0 \text { in } S, \\
\nabla \psi \cdot \hat{\mathbf{n}}=0 \text { on } \eta=0 .
\end{gathered}
$$

A further expansion of this equation yields 


$$
\begin{gathered}
\left(\frac{1}{k^{2}} \Delta+\frac{i}{k} \frac{2 V}{1-\kappa \eta} \partial_{s}+\frac{i}{k} \frac{V_{s}}{1-\kappa \eta}+1-V^{2}\right) \psi=0 \text { in } S, \\
\psi_{\eta}=0 \text { on } \eta=0,
\end{gathered}
$$

where

$$
V(\eta, s)=\frac{h \eta(1-\kappa \eta / 2)+\beta}{1-\kappa \eta}
$$

So far we have simply rewritten the linearized problem (2.9)-(2.10) in terms of the function $\psi$ (introduced via the changes of variables (2.19)) and in terms of the independent variables $s$ and $\eta$ to obtain a convenient formulation of the problem valid near $\partial \Omega$. At this point, we wish to begin a process of formal matched asymptotic expansion (based on the assumption $k \gg 1$ ) by introducing stretched coordinates to solve a boundary layer problem which will then be matched to the normal state in the interior.

As we are seeking a boundary layer solution and we have a factor of $\left(1 / k^{2}\right)$ multiplying the Laplacian in (2.22) it is natural to seek a solution which depends not on the normal variable $\eta$ but on the stretched variable

$$
\xi=k \eta
$$

What is not so obvious, but nonetheless necessary, is that we must stretch the tangential variable as well, by introducing

$$
\tau=k^{1 / 4}\left(s-s_{0}\right)
$$

Here $s_{0}$ is a value of arc length along the boundary which remains to be determined. The transverse length scale $\left(1 / k^{1 / 4}\right)$ represents a balance between the quadratic nature of the curvature at the maximum and the transverse modulation of the amplitude, a fact best explained a posteriori. Putting it another way, any expansion which fails to incorporate this stretched tangential dependence - that is, which fails to sufficiently promote the appearance of tangential derivatives of the solution as we expand-will lead to inconsistencies later in the asymptotic analysis.

Once one resolves to seek a solution depending on $\xi$ and $\tau$, however, one is obligated to pursue a solution which is not only localized near $\partial \Omega$, but localized near the (so far arbitrary) point corresponding to $s=s_{0}$ as well. Accordingly, this solution must be matched to the normal state both in the interior of $\Omega$ ( $\xi$ large) and along the boundary away from $s=s_{0}(|\tau|$ large).

Thus, we assume an expansion for $\psi$ of the form

$$
\psi=\psi_{0}(\xi, \tau)+\frac{1}{k^{1 / 4}} \psi_{1}(\xi, \tau)+\frac{1}{k^{1 / 2}} \psi_{2}(\xi, \tau)+\frac{1}{k^{3 / 4}} \psi_{3}(\xi, \tau)+\cdots
$$

and an expansion for $h=h(k)$ of the form

$$
h=h_{0} k+h_{1} k^{3 / 4}+h_{2} k^{1 / 2}+h_{3} k^{1 / 4}+h_{4}+\cdots .
$$

To carry out the analysis, we need an expansion for the expression $V$ in powers of $k$. To this end, we first express the curvature $\kappa$ as a function of $\tau$, say $K(\tau)$, and Taylor expand about $s=s_{0}$ using (2.26) to obtain

$$
K(\tau)=\kappa\left(s_{0}\right)+\frac{1}{k^{1 / 4}} \kappa_{s}\left(s_{0}\right) \tau+\frac{1}{k^{1 / 2}} \kappa_{s s}\left(s_{0}\right) \frac{\tau^{2}}{2}+\mathcal{O}\left(\frac{1}{k^{3 / 4}}\right)=\bar{\kappa}+\frac{1}{k^{1 / 4}} \bar{\kappa}_{s} \tau+\frac{1}{k^{1 / 2}} \bar{\kappa}_{s s} \frac{\tau^{2}}{2}+\mathcal{O}\left(\frac{1}{k^{3 / 4}}\right),
$$

where $\bar{\kappa}, \bar{\kappa}_{s}$, and $\bar{\kappa}_{s s}$ denote $\kappa\left(s_{0}\right), \kappa_{s}\left(s_{0}\right)$, and $\kappa_{s s}\left(s_{0}\right)$, respectively.

To expand $V$, we substitute (2.25) and (2.29) in (2.24) to find 


$$
\begin{aligned}
V= & \left(h_{0} \xi+\beta\right)+\frac{1}{k^{1 / 4}}\left(h_{1} \xi\right)+\frac{1}{k^{1 / 2}}\left(h_{2} \xi\right)+\frac{1}{k^{3 / 4}}\left(h_{3} \xi\right) \\
& +\frac{1}{k}\left\{\left(h_{4} \xi+\left(\frac{h_{0}}{2} \xi^{2}+\beta \xi\right) \bar{\kappa}\right\}+\frac{1}{k^{5 / 4}}\left\{\left(h_{5} \xi+\frac{h_{1}}{2} \xi^{2} \bar{\kappa}+\left(\frac{h_{0}}{2} \xi^{2}+\beta \xi\right) \bar{\kappa}_{s} \tau\right\}\right.\right. \\
& +\frac{1}{k^{3 / 2}}\left\{\left(h_{6} \xi+\frac{h_{2}}{2} \xi^{2} \bar{\kappa}+\frac{h_{1}}{2} \xi^{2} \bar{\kappa}_{s} \tau+\left(\frac{h_{0}}{2} \xi^{2}+\beta \xi\right) \bar{\kappa}_{s s} \frac{\tau^{2}}{2}\right\}+\mathcal{O}\left(\frac{1}{k^{7 / 4}}\right) .\right.
\end{aligned}
$$

Using (2.12) and (2.27), we expand the Laplacian

$$
\frac{1}{k^{2}} \Delta=\partial_{\xi \xi}^{2}-\frac{1}{k} \bar{\kappa} \partial_{\xi}-\frac{1}{k^{5 / 4}} \bar{\kappa}_{s} \tau \partial_{\xi}+\frac{1}{k^{3 / 2}}\left\{\partial_{\tau \tau}-\bar{\kappa}_{s s} \frac{\tau^{2}}{2} \partial_{\xi}\right\}+\mathcal{O}\left(\frac{1}{k^{7 / 4}}\right) .
$$

The imaginary terms in (2.22) can be expanded using (2.13), (2.25) and (2.26)

$$
\frac{i}{k} \frac{2 V}{1-\kappa \eta} \partial_{s}+\frac{i}{k} \frac{V_{s}}{1-\kappa \eta}=\frac{i}{k^{3 / 4}} 2 V \partial_{\tau}+\frac{i}{k^{3 / 4}} V_{\tau}+\mathcal{O}\left(\frac{1}{k^{7 / 4}}\right) .
$$

We can now proceed to expand and solve for successive orders in the expansion for $\psi$.

\section{A. Leading order balance: The half-plane problem}

The basis for our expansion is that the leading-order balance in the problem is exactly equivalent to the instability problem for the half-plane. This problem was first considered by Saint-James and de Gennes, ${ }^{2}$ and yields an exponentially localized superconducting region near the boundary. In the general domain we will construct a solution whose leading order structure is equivalent to the solution of the half-plane problem with an amplitude that varies along the boundary.

We can now expand the problem at leading order ( $($ (1)); Eqs. (2.22) and (2.23) yield

$$
\begin{gathered}
\psi_{0_{\xi \xi}}+\left(1-\left(h_{0} \xi+\beta\right)^{2}\right) \psi_{0}=0 \text { for } 0<\xi<\infty, \\
\psi_{0_{\xi}}(0)=0, \quad \psi_{0_{\xi}}(\infty)=0,
\end{gathered}
$$

where the second boundary condition allows us to match the boundary layer solution to the normal state in the interior of $\Omega$. This is exactly the half-plane problem considered formally in Refs. 2 and 3 , and treated rigorously in Refs. 6 and 7.

Recall that we seek the first appearance of a nonzero state as the magnetic field is decreased. Hence we view (2.33) as a double eigenvalue problem for $h_{0}$ and $\beta$ in which $\beta$ is to be determined so as to yield $\bar{h}$, the largest possible value of $h_{0}$. Note that $\beta$ corresponds to a transverse (i.e., parallel to the boundary) wave number, which in the half-plane problem arises naturally through separation of variables. We now tabulate a set of results known for this problem, which will form the basis for our perturbation expansion. With the exception of identities (2.34)-(2.36) below, the proof of the Lemma can be found, for example, in Ref. 15. See also Refs. 6 and 7. The derivation of (2.34)-(2.36) can be found in the appendix.

Lemma: There exists a largest value of $h_{0}(\beta)$, denoted by $\bar{h}$, for which (2.33) possesses a nontrivial solution. This occurs when $\beta$ takes the value -1 . The corresponding eigenfunction, denoted henceforth by $\bar{\psi}(\xi)$, satisfies

$$
\begin{aligned}
& \mathscr{L} \bar{\psi}=0 \quad \text { for } \quad 0<\xi<\infty, \\
& \bar{\psi}_{\xi}(0)=0, \quad \bar{\psi}_{\xi}(\infty)=0,
\end{aligned}
$$

with the normalization $\bar{\psi}(0)=1$.

The operator $\mathscr{B}$ is defined by

$$
\mathscr{B}(\cdot) \equiv \partial_{\xi \xi}(\cdot)+\left(1-(\bar{h} \xi-1)^{2}\right)(\cdot),
$$


and is self-adjoint in the $L^{2}$ inner-product.

The solution $\bar{\psi}$ decays exponentially as $\xi \rightarrow \infty$ and, letting $J_{0} \equiv \int_{0}^{\infty} \bar{\psi}^{2} d \xi$, we have that $\bar{\psi}$ satisfies the following identities:

$$
\begin{gathered}
J_{1} \equiv \int_{0}^{\infty} \xi \bar{\psi}^{2} d \xi=\frac{1}{\bar{h}} J_{0}, \\
J_{2} \equiv \int_{0}^{\infty} \xi^{2} \bar{\psi}^{2} d \xi=\frac{3}{2 \bar{h}^{2}} J_{0}, \\
J_{3} \equiv \int_{0}^{\infty} \xi^{3} \bar{\psi}^{2} d \xi=\frac{1}{6 \bar{h}^{2}}+\frac{5}{2 \bar{h}^{3}} J_{0} .
\end{gathered}
$$

Remark: Through numerical approximation one finds $\bar{h}=1.69461+$ and $J_{0}=1.00784+$.

In light of the absence of any $\tau$ dependence in (2.33), we may write

$$
\psi_{0}(\xi, \tau)=W_{0}(\tau) \bar{\psi}(\xi)
$$

where $W_{0}(\tau)$ is a (perhaps complex) amplitude corresponding to a transverse modulation of $\bar{\psi}(\xi)$ on the $\tau$ length scale whose behavior will be determined at higher order. This type of modulation or multiple scale expansion is familiar from many physical systems. ${ }^{16}$

\section{B. Higher order corrections}

At orders $\mathscr{O}\left(1 / k^{1 / 4}\right)$ and $\mathscr{O}\left(1 / k^{1 / 2}\right)$ we find

$$
\begin{gathered}
\mathscr{B}\left(\psi_{i}\right)=2 h_{i} \xi(\bar{h} \xi-1) \bar{\psi}(\xi) W_{0}(\tau) \quad \text { for } \quad i=1,2, \\
\left(\psi_{i}\right)_{\xi}(0)=0, \quad\left(\psi_{i}\right)_{\xi}(\infty)=0 .
\end{gathered}
$$

If we define the $L^{2}$ inner-product,

$$
\langle\psi, \phi\rangle=\int_{0}^{\infty} \psi^{*} \phi d \xi
$$

then the Fredholm Alternative Theorem yields necessary and sufficient solvability conditions for (2.37). That is, orthogonality to $\bar{\psi}$,

$$
\left\langle\bar{\psi}, \mathscr{L}\left(\psi_{i}\right)\right\rangle=0 \quad \text { for } \quad i=1,2,
$$

implies

$$
h_{i} W_{0} \frac{J_{0}}{\bar{h}}=0 .
$$

Thus we conclude $h_{1}=h_{2}=0$ so that $\mathscr{L}\left(\psi_{i}\right)=0$ and we have

$$
\psi_{1}=W_{1}(\tau) \bar{\psi}(\xi) \quad \text { and } \quad \psi_{2}=W_{2}(\tau) \bar{\psi}(\xi)
$$

with the functions $W_{1}$ and $W_{2}$ yet to be determined.

Proceeding to $\mathscr{Q}\left(1 / k^{3 / 4}\right)$,

$$
\begin{gathered}
\mathscr{L}\left(\psi_{3}\right)=2 h_{3} \xi(\bar{h} \xi-1) \bar{\psi} W_{0}-2 i(\bar{h} \xi-1) \bar{\psi} W_{0 \tau} \quad \text { for } \quad 0<\xi<\infty, \\
\psi_{3_{\xi}}(0)=0, \quad \psi_{3_{\xi}}(\infty)=0 .
\end{gathered}
$$


Application of the solvability condition at this order yields

$$
\left\langle\bar{\psi}, \mathscr{L}\left(\psi_{3}\right)\right\rangle=0=h_{3} W_{0} \frac{J_{0}}{\bar{h}}
$$

hence $h_{3}=0$. Now note that since $\partial_{\xi}(\mathscr{B}(\bar{\psi}))=0$, one has

$$
\mathscr{L}\left(\bar{\psi}_{\xi}\right)=2 \bar{h}(\bar{h} \xi-1) \bar{\psi} .
$$

One also readily verifies that $\bar{\psi}_{\xi \xi}(0)=\bar{\psi}_{\xi \xi}(\infty)=0$ (recall that in fact $\bar{\psi}$ decays exponentially at infinity) so that we find

$$
\psi_{1}=W_{3}(\tau) \bar{\psi}(\xi)-\frac{i}{\bar{h}} \bar{\psi}_{\xi} W_{0}
$$

where $W_{3}$ remains to be determined.

We now proceed to order $\odot(1 / k)$,

$$
\begin{gathered}
\mathscr{L}\left(\psi_{4}\right)=h_{4}[2 \xi(\bar{h} \xi-1) \bar{\psi}] W_{0}+\bar{\kappa}\left[\xi(\bar{h} \xi-1)(\bar{h} \xi-2) \bar{\psi}+\bar{\psi}_{\xi}\right] W_{0}-[2 i(\bar{h} \xi-1) \bar{\psi}] W_{1_{\tau}}, \\
\psi_{4_{\xi}}(0)=0, \quad \psi_{4_{\xi}}(\infty)=0 .
\end{gathered}
$$

Solvability at this order takes the form,

$$
\left(h_{4} \frac{J_{0}}{\bar{h}}-\frac{\bar{\kappa}}{3}\right) W_{0}=0,
$$

where we have applied the identities (2.34)-(2.35), and integration by parts. Thus

$$
h_{4}=\frac{\bar{h}}{3 J_{0}} \bar{\kappa}=\frac{\bar{h}}{3 J_{0}} \kappa\left(s_{0}\right) .
$$

Note that we seek the largest value of $h$ (hence of $h_{4}$ ) at which a superconducting solution emerges; clearly we can maximize $h_{4}$ by choosing to expand around the point where $\kappa\left(s_{0}\right)$ is a maximum, i.e., by choosing $s_{0}=0$. Consequently, we conclude that instability first occurs at the point of maximum curvature, so that

$$
\begin{gathered}
\bar{\kappa}=\max _{s_{0}} \kappa\left(s_{0}\right)=\kappa(0), \\
\bar{\kappa}_{s}=0, \\
\bar{\kappa}_{s s} \leqslant 0 .
\end{gathered}
$$

We shall make the (generic) assumption that

$$
\bar{\kappa}_{s s}<0 \text {. }
$$

We have also tacitly assumed that the curvature reaches a unique maximum; in the case of multiple isolated maximums (such as an ellipse), we could construct an exponentially localized solution at each which would interact only exponentially weakly. In the case when the second derivative vanishes at the maximum (such as the disc), our scaling ansatz will prove to be invalid at higher order.

In addition, formula (2.43) — specifically the fact that $h_{4}$ is positive-bears the information that onset of superconductivity in a bounded sample occurs at a higher value of applied field than for the case where $\Omega$ is a half-space. This correction agrees with that computed by Bauman et al. ${ }^{10}$ for the disc. 
We could proceed to solve for $\psi_{4}$ at this point (an explicit solution can be obtained via variation of parameters), however we omit this calculation as it will play no further role in our analysis.

We proceed to the next order and collect terms of $\mathscr{O}\left(1 / k^{5 / 4}\right)$,

$$
\begin{gathered}
\mathscr{L}\left(\psi_{5}\right)=h_{5}[2 \xi(\bar{h} \xi-1) \bar{\psi}] W_{0}+h_{4}[2 \xi(\bar{h} \xi-1) \bar{\psi}] W_{1} \\
+\bar{\kappa}\left[\xi(\bar{h} \xi-1)(\bar{h} \xi-2) \bar{\psi}+\bar{\psi}_{\xi}\right] W_{1}-[2 i(\bar{h} \xi-1) \bar{\psi}] W_{2_{\tau}} \\
\psi_{5_{\bar{\xi}}}(0)=0, \quad \psi_{5_{\xi}}(\infty)=0 .
\end{gathered}
$$

Once again, integration against $\bar{\psi}$ yields the solvability condition. The terms in $W_{1}$ and $W_{2}$ in the solvability condition vanish in an analogous fashion to those in $W_{0}$ and $W_{1_{\tau}}$ at the previous order (taking into account the value of $h_{4}$ determined by (2.43)). Hence, $h_{5}=0$. Once again, we neglect the calculation of $\psi_{5}$ as it plays no further role in our analysis.

Finally, we proceed to the next order and collect terms of $\mathscr{O}\left(1 / k^{3 / 2}\right)$,

$$
\begin{gathered}
\mathscr{L}\left(\psi_{6}\right)=h_{6}[2 \xi(\bar{h} \xi-1) \bar{\psi}] W_{0}+\bar{\kappa}_{s s} \frac{\tau^{2}}{2}\left[\xi(\bar{h} \xi-1)(\bar{h} \xi-2) \bar{\psi}+\bar{\psi}_{\xi}\right] W_{0}-\left[\frac{2}{\bar{h}}(\bar{h} \xi-1) \bar{\psi}_{\xi}+\bar{\psi}\right] W_{0_{\tau \tau}} \\
+h_{4}[2 \xi(\bar{h} \xi-1) \bar{\psi}] W_{2}+\bar{\kappa}\left[\xi(\bar{h} \xi-1)(\bar{h} \xi-2) \bar{\psi}+\bar{\psi}_{\xi}\right] W_{2}-[2 i(\bar{h} \xi-1) \bar{\psi}] W_{3_{\tau}}, \\
\psi_{\sigma_{\xi}}(0)=0, \quad \psi_{\sigma_{\xi}}(\infty)=0 .
\end{gathered}
$$

Once again, we integrate against $\bar{\psi}$. The terms proportional to $W_{2}$ and $W_{3_{\tau}}$ in the solvability condition vanish in an analogous fashion to those proportional to $W_{1}$ and $W_{2_{\tau}}$ respectively at the previous order. However, we obtain a nontrivial solvability condition for $W_{0}(\tau)$. Applying the identities (2.34)-(2.36) and integrating by parts yields

$$
-\frac{1}{\bar{h}} W_{0_{\tau \tau}}+\left(h_{6} \frac{J_{0}}{\bar{h}}-\frac{\bar{\kappa}_{s s}}{6} \tau^{2}\right) W_{0}=0 .
$$

At this point we see the motivation for the introduction of the $\tau$ lengthscale; on this length scale the tranverse variation of the amplitude $\left(W_{0_{\tau \tau}}\right)$ balances the quadratic variation of the curvature $\left(\bar{\kappa}_{s s} \tau^{2} / 2\right)$.

Boundary conditions on $W_{0}(\tau)$ are determined by a need to match to the normal state at large $|\tau|$, for which $\Psi^{(1)}$ and consequently $W_{0}$ vanish. Thus, we apply the condition that $W_{0}(\tau)$ vanishes in the far-field,

$$
W_{0}(\tau) \rightarrow 0 \quad \text { as }|\tau| \rightarrow \infty
$$

with the understanding that $\tau$ is in the matching region, $1 \ll|\tau| \ll k^{1 / 4}$. Fortunately, $W_{0}(\tau)$ decays exponentially and consequently this matching will only introduce exponentially small corrections in the expansion.

Fortuitously, Eq. (2.46) is real, and consequently admits real solutions for $W_{0}$ (we expect $W_{n}$ to be complex in general). We can rescale (2.46) into the standard form for the harmonic oscillator equation; define

$$
\zeta=\alpha \tau, \quad w(\zeta)=W_{0}(\zeta / \alpha), \quad \lambda=-\frac{J_{0} h_{6}}{\alpha^{2}}
$$

where

$$
\alpha=\left(\frac{-2 \bar{\kappa}_{s s} \bar{h}}{3}\right)^{1 / 4}
$$


remembering that at the maximum of curvature, we have $\bar{\kappa}_{s s}<0$. Equations (2.46) and (2.47) become

$$
w_{\zeta \zeta}+\left(\lambda-\frac{1}{4} \zeta^{2}\right) w=0, \quad w(\zeta) \rightarrow 0 \quad \text { as } \quad|\zeta| \rightarrow \infty
$$

The eigenvalues and eigenfunctions of this problem are given by

$$
\lambda_{n}=n+\frac{1}{2}, \quad w_{n}(\zeta)=\operatorname{He}_{n}(\zeta) e^{-\zeta^{2} / 4}, \quad n=0,1,2,3, \ldots
$$

where $\mathrm{He}_{n}(\zeta)$ are the Hermite polynomials (cf. Ref. 17). Note that the smallest eigenvalue is $\lambda_{0}=1 / 2$, with corresponding eigenfunction $w_{0}(\zeta)=e^{-\zeta^{2} / 4}$. Hence, the largest (least negative) eigenvalue for (2.46) is given by

$$
h_{6}=-\frac{1}{2 J_{0}}\left(\frac{-2 \bar{\kappa}_{s s} \bar{h}}{3}\right)^{1 / 2} \text {, }
$$

and the corresponding eigenfunction is

$$
W_{0}(\tau)=e^{-\alpha^{2} \tau^{2} / 4}
$$

The exponential decay of $W_{0}$ away from $\tau=0$ reveals that the phenomenon of onset is highly localized near the point of maximum curvature for $\partial \Omega$. We have now completely determined the leading order solution for $\Psi^{(1)}$ to the linearized problem (2.9), (2.10) in the asymptotic regime $\kappa \gg 1$.

\section{DISCUSSION}

In this paper we have examined the instability of the normal state to superconductivity with decreasing magnetic field for a closed smooth cylindrical region subject to a vertical magnetic field. We have examined the problem asymptotically in the boundary layer limit $(k \gg 1)$. Instability first occurs in a region exponentially localized near the point of maximum curvature on the boundary. The transition occurs at a value of the magnetic field,

$$
H_{c_{3}}=\bar{h} k+\frac{\bar{h}}{3 J_{0}} \bar{\kappa}-\frac{1}{2 J_{0}}\left(\frac{-2 \bar{\kappa}_{s s} \bar{h}}{3}\right)^{1 / 2} \frac{1}{k^{1 / 2}}+\mathcal{O}\left(\frac{1}{k}\right),
$$

where $\bar{h} k$ is the transition value associated with the half-plane (cf. Refs. 2 and 3), the second term is a positive constant times the maximum curvature $(\bar{\kappa})$ that agrees with the correction computed for the disc (cf. Ref. 10), the third term is a negative correction of order $k^{-1 / 2}$, associated with the quadratic variation of the curvature at the maximum, and the error term follows from continuing the expansion documented in the previous section.

At leading order, the superconducting order-parameter satisfies

$$
\left|\Psi^{(1)}\right| \sim \bar{\psi}(\xi) e^{-\alpha^{2} \tau^{2} / 4}
$$

where

$$
\xi=k \eta, \quad \tau=k^{1 / 4} s, \quad \text { and } \quad \alpha=\left(\frac{-2 \bar{\kappa}_{s s} \bar{h}}{3}\right)^{1 / 4}
$$

Here $\bar{\psi}$ is the profile associated with transition in the half-plane problem-it is an exponentially decaying function of the normal distance to the boundary $(\eta)$ scaled by the boundary layer thickness $(1 / k)$. The amplitude along the boundary is modulated by a Gaussian, $e^{-\alpha^{2} \tau^{2} / 4}$, where $\tau$ 
is the arclength measured from the point of maximum curvature (at $s=0)$ scaled by a length scale intermediate between the boundary layer thickness and the characteristic length scale of the domain (unity).

Physically, this result is a refinement of the corresponding results for the half-plane and the disc. The half-plane result yields the leading order transition value for the magnetic field and exponentially decaying boundary layer profile, $k \bar{h}$ and $\bar{\psi}$, respectively. The disc calculation shows that transition occurs at a higher value of the magnetic field where the correction is of order unity and proportional to the curvature (i.e., the inverse of the radius). In contrast to the half-plane and disc calculation where onset occurs along the entire boundary simultaneously, in a general domain the profile is exponentially localized near the point of maximum curvature on the boundary on the $\tau$ length scale; the transition value is slightly below that of the oscillating disc at the point of maximum curvature.

An additional effect of this exponential localization of the transition profile is that the periodicity of the boundary apparently has an exponentially small effect on the critical magnetic field and the various eigenmodes are never degenerate. This is in sharp contrast to the theoretical calculation for the disc where periodicity "quantizes" the transverse wave numbers and at various critical parameter values there is a transition between different modes as the most unstable. We note that this quantization in a disc is captured via formal matched asymptotics in Ref. 18. These characteristics are also observed experimentally. ${ }^{19,20}$ We suggest here that this degeneracy occurs only in the presence of symmetry in the sample geometry.

Finally, we note that the approach here is quite general, and should allow for the incorporation of nonlinearity, temporal evolution through relaxational effects, etc. The result we believe will be a Newell-Whitehead-Segal-type equation (cf. Ref. 16),

$$
W_{T}=W_{S S}+[h-\kappa(S)] W-W|W|^{2},
$$

where $W$ is a transverse modulation amplitude of the boundary layer profile, $S$ is a scaled arc length, $T$ is a slow time scale associated with relaxational effects (cf. Ref. 4), $h$ is a scaled magnetic field, and $\kappa(S)$ measures curvature variation along the boundary. A model of this sort could be used to study hysteresis, symmetry breaking and a host of other phenomena which may lead to better understanding and prediction of the behavior of superconductors.

Note added in proof: After completing our work we learned about Reference 21.

\section{ACKNOWLEDGMENTS}

The authors gratefully acknowledge fruitful discussions with Joe Keller, John Neu, and Jacob Rubinstein on the topic of this paper. A.J.B. wishes to thank the MSRI and P.S. wishes to thank the Department of Mathematics at Stanford University for the hospitality extended during visits covering the period in which this research was conducted.

The work of P.S. was supported by the National Science Foundation under Grants Nos. DMS-9322617 and DMS-9705774 and by a U.S.-Israel Bi-National Grant.

\section{APPENDIX: DERIVATION OF MOMENT IDENTITIES (2.34)-(2.36)}

To derive identities $(2.34)-(2.36)$, we observe that the operator $\mathscr{L}$ maps the vector space of functions of the form $a(\xi) \bar{\psi}+b(\xi) \bar{\psi}_{\xi}$, where $a(\xi)$ and $b(\xi)$ are polynomials, into itself. A little algebra shows that for a polynomial $c(\xi)$,

$$
\mathscr{B}\left(2 c \bar{\psi}_{\xi}-c_{\xi} \bar{\psi}\right)=-\left\{c_{\xi \xi \xi}+4 \bar{h} \xi(2-\bar{h} \xi) c_{\xi}+4 \bar{h}(1-\bar{h} \xi) c\right\} \bar{\psi} .
$$

Multiplying by $\bar{\psi}$, integrating over the range and applying integration by parts, we reach the identity

$$
\int_{0}^{\infty} \bar{\psi} \mathscr{L}\left(2 c \bar{\psi}_{\xi}-c_{\xi} \bar{\psi}\right) d \xi=c_{\xi \xi}(0) .
$$

If we now define the moments, 


$$
J_{n} \equiv \int_{0}^{\infty} \xi^{n} \bar{\psi}^{2} d \xi
$$

we can derive a recursion relationship for $J_{n}$ by choosing $c=\xi^{n}$, substituting into (A1) and applying the identity (A2)

$$
\begin{gathered}
c=1: \quad 0=-4 \bar{h} J_{0}+4 \bar{h}^{2} J_{1}, \\
c=\xi: \quad 0=-12 \bar{h} J_{1}+8 \bar{h}^{2} J_{2}, \\
c=\xi^{2}: \quad 2=-20 \bar{h} J_{2}+12 \bar{h}^{2} J_{3}, \\
c=\xi^{n}: \quad 0=-n(n-1)(n-2) J_{n-3}-4 \bar{h}(2 n+1) J_{n}+4(n+1) \bar{h}^{2} J_{n+1} \quad n \geqslant 3 .
\end{gathered}
$$

The identities (2.34)-(2.36) and expressions for $J_{n}$ in terms of $J_{0}$ can now be found by solving these relations.

${ }^{1}$ V. L. Ginzburg and L. D. Landau, "On the theory of superconductivity," JETP 20, 1064-1082 (1950).

${ }^{2}$ D. Saint-James and P. G. de Gennes, "Onset of superconductivity in decreasing fields," Phys. Lett. 7, 306-308 (1963).

${ }^{3}$ S. J. Chapman, "Nucleation of superconductivity in decreasing fields, I," Eur. J. Appl. Math. 5, 449-468 (1994).

${ }^{4}$ S. J. Chapman, “Nucleation of superconductivity in decreasing fields, II,' Eur. J. Appl. Math. 5, 469-494 (1994).

${ }^{5}$ M. H. Millman and J. B. Keller, "Perturbation theory of nonlinear boundary value problems," J. Math. Phys. 10, 342-361 (1969).

${ }^{6} \mathrm{C}$. Bolley, "Modélisation de champ de retard à la condensation d'un supraconducteur par un problème de bifurcation," $M^{2}$ AN 26, 235-287 (1992)

${ }^{7}$ C. Bolley and B. Helffer "An application of semi-classical analysis to the asymptotic study of the supercooling field of a superconducting material,"’ Ann. Inst. Henri Poincaré: Phys. Theor. 58, 189-233 (1993).

${ }^{8}$ F. Odeh, "Existence and bifurcation theorems for the Ginzburg-Landau equations," J. Math. Phys. 8, 2351-2357 (1967).

${ }^{9}$ T. Giorgi, and D. Phillips, "The breakdown of superconductivity due to strong fields for the Ginzburg Landau model" (preprint)

${ }^{10} \mathrm{P}$. Bauman, D. Phillips, and Q. Tang, "Stable nucleation for the Ginzburg Landau system with an applied magnetic field," Arch. Ration. Mech. Anal. (to be published).

${ }^{11}$ S. J. Chapman, "Nucleation of vortices in type-II superconductors in increasing magnetic fields," Appl. Math. Lett. 10 29-31 (1997).

${ }^{12}$ Q. Du, M. D. Gunzburger, J. S. Peterson, "Analysis and approximation of the Ginzburg Landau model of superconductivity,” SIAM (Soc. Ind. Appl. Math.) Rev. 34, 45-81 (1992).

${ }^{13}$ R. Aris, Vectors, Tensors and the Basic Equations of Fluid Mechanics (Prentice-Hall Englewood Cliffs, 1962).

${ }^{14}$ D. Sarocka and A. Bernoff, "An intrinsic equation of interfacial motion for the solidification of a pure hypercooled melt," Physica D 85, 348-374 (1995).

${ }^{15}$ M. Dauge and B. Helffer "Eigenvalues variation I, Neumann problem for Sturm-Liouville operators," J.D.E. 104 243-262 (1993).

${ }^{16}$ M. C. Cross and P. C. Hohenberg, “Pattern formation outside of equilibrium,” Rev. Mod. Phys. 65, 854-1112 (1993).

${ }^{17}$ M. Abramowitz and I. A. Stegun Handbook of Mathematical Functions (Dover, New York, (1964).

${ }^{18}$ J. Rubinstein and P. Sternberg "Onset of superconductivity in a disc" (in preparation).

${ }^{19}$ O. Buisson, P. Gandit, R. Rammal, Y. Y. Wang, and B. Pannetier, "Magnetization oscillations of a superconducting disk," Phys. Lett. A A 150, 36-42 (1990).

${ }^{20}$ V. V. Moschchalkov, L. Gielen, C. Strunk, R. Jonckheere, X. Qiu, C. Van Haesendonck, and Y. Bruynseraede, "Effect of sample topology on the critical fields of mesoscopic superconductors," Nature (London) 373, 319-322 (1995).

${ }^{21}$ K. Lu and X. B. Pan, "Estimates of upper critical external magnetic field for the Ginzburg-Landau equation," preprint. 